\title{
Electromagnetic Aharonov-Bohm effect in a two-dimensional electron gas ring
}

\author{
W. G. van der Wiel, ${ }^{1,2}$ Yu. V. Nazarov, ${ }^{2}$ S. De Franceschi, ${ }^{2, *}$ T. Fujisawa, ${ }^{3}$ J. M. Elzerman, ${ }^{2, *}$ E. W. G. M. Huizeling, ${ }^{2}$ \\ S. Tarucha, ${ }^{3,4, *}$ and L. P. Kouwenhoven ${ }^{1,2, *}$ \\ ${ }^{1}$ PRESTO, Japan Science and Technology Corporation (JST), Department of Physics, University of Tokyo, \\ 7-3-1, Hongo, Bunkyo-ku, Tokyo, 113-0033, Japan \\ ${ }^{2}$ Department of NanoScience and DIMES, Delft University of Technology, PO Box 5046, 2600GA Delft, The Netherlands \\ ${ }^{3}$ NTT Basic Research Laboratories, NTT Corporation, 3-1 Morinosato-Wakamiya, Atsugi-shi, Kanagawa 243-0198, Japan \\ ${ }^{4}$ Department of Physics, University of Tokyo, 7-3-1, Hongo, Bunkyo-ku, Tokyo 113-0033, Japan
}

(Received 13 June 2002; published 29 January 2003)

\begin{abstract}
We define a mesoscopic ring in a two-dimensional electron gas interrupted by two tunnel barriers, enabling us to apply a well-defined potential difference between the two halves of the ring. The electron interference in the ring is modified using a perpendicular magnetic field and a bias voltage. We observe clear Aharonov-Bohm oscillations up to the quantum Hall regime as a function of both parameters. The electron travel time between the barriers is found to increase with the applied magnetic field. Introducing a scattering model, we develop a method to measure the nonequilibrium electron dephasing time, which becomes very short at high voltages and magnetic fields. The relevance of electron-electron interactions is discussed.
\end{abstract}

DOI: 10.1103/PhysRevB.67.033307 PACS number(s): 73.23.-b, 03.65.Ta, 72.10.Bg, 72.20.My

Aharonov and Bohm predicted that the phase of an electron wave is affected by both magnetic and electric potentials, being observable in an interference experiment. ${ }^{1} \mathrm{~A}$ magnetic flux, $\Phi$, threading a loop leads to an oscillating contribution to the conductance with period $\Phi_{0}=h / e$ : the magnetic Aharonov-Bohm (AB) effect. In a solid state device, $\mathrm{AB}$ oscillations are observable at mesoscopic scale, where quantum coherence is preserved. The effect was first observed in a metal ring $^{2}$ and later in two-dimensional electron gas (2DEG) rings. ${ }^{3}$

In the case of the electrostatic $\mathrm{AB}$ effect, as originally suggested, the electron phase is affected by an electrostatic potential, although the electrons do not experience an electric field. ${ }^{1}$ The required geometry is difficult to realize and so far only geometries have been investigated where an electric field changes the interference pattern. ${ }^{4-7}$ In Ref. 8 a ring geometry interrupted by tunnel barriers was suggested, where a bias voltage, $V$, leads to an electrostatically controlled $\mathrm{AB}$ effect with predictions very similar to the original proposal. ${ }^{1}$ This effect was observed in a disordered metal ring with two tunnel junctions. ${ }^{9}$

In this Brief Report, we report the observation of an electrostatic $A B$ effect in a quasiballistic ring-shaped 2DEG system interrupted by tunnel barriers. There are two important distinctions from metal systems that play a key role in the present study. First, only a few electron modes are involved in transport, in contrast to tens of thousands in a typical metal wire. Second, in a $2 \mathrm{DEG}$ we can enter the quantum Hall regime using a perpendicular magnetic field, $B$.

We observe both a magnetic and electrostatic AB effect up to the edge channel regime (filling factor $\nu=3$ ). The electron travel time between the barriers increases with $B$. We ascribe this to the bending effect of the Lorentz force on the electron trajectories. Importantly, the electrostatic oscillations form a new tool to determine the nonequilibrium electron dephasing time, $\tau_{\phi}$. The possibility to estimate the electron dephasing time at a controlled, finite energy and magnetic field distinguishes this method from weak localiza- tion experiments used to estimate the (equilibrium) electron dephasing time. ${ }^{10}$ We find that $\tau_{\phi}$ decreases as a function of $B$ and $V$ and reaches $\tau_{\phi}|\varepsilon| \simeq \hbar$ at the highest $B$, with $\varepsilon$ the electron energy measured from the Fermi level. This demonstrates the increasing importance of many-body effects at quantizing magnetic fields where the Fermi-liquid picture and the quasiparticle concept are at the edge of applicability. Measurements of the inelastic relaxation rate in a disordered Fermi liquid have been reported in Refs. 11 and 12.

Our device [Fig. 1(a)] consists of an AB ring defined in a 2DEG. ${ }^{13}$ The gate electrodes $V_{g u}$ and $V_{g l}$ define a barrier in each arm of the ring. The effective width of the arms supports $N_{\mathrm{tr}} \simeq 10$ transport channels (i.e., the conductance of the ring without barriers is approximately $10 e^{2} / h$ at $\left.B=0 \mathrm{~T}\right)$. In addition to the dc bias voltage, $V$, we apply a relatively small ac voltage ( 3 to $10 \mu \mathrm{V}$ ) between source and drain contacts. We measure the differential conductance, $G=d I / d V$, using a lock-in technique in a dilution refrigerator at the base temperature of $15 \mathrm{mK}$. The experimental results presented in this Brief Report are obtained on one sample, but are supported by extensive results in the three devices we studied. In addition, the main features are reproduced after warming up and cooling down samples.

Figure 1(c) shows the differential $\mathrm{AB}$ conductance, $G_{A B}$, versus $B$ and $V$, around 1.9 T. $G_{A B}$ is extracted from the measured $G$ by fitting a polynomial to the smoothly varying background in $G$. Subtraction of this polynomial yields the oscillating part, $G_{A B}$. The barriers are tuned such that the conductance through one arm is $0.2 e^{2} / h . G_{A B}$ as function of magnetic field exhibits $\mathrm{AB}$ oscillations with a period $B_{0}$ $=1.0 \mathrm{mT}$, in good agreement with $h /(e S)$, where $S$ is the area enclosed by the ring. The effect of the dc bias voltage, $V$, is schematically shown in Fig. 1(b). The transmitted part of the electron wave has energy $\varepsilon_{R}$, whereas the reflected part has $\varepsilon_{L}$ with respect to the Fermi level in each half of the ring (note $e V=\varepsilon_{R}-\varepsilon_{L}$ and $\left|\varepsilon_{L}\right|,\left|\varepsilon_{R}\right|,|e V| \ll E_{F}$ ). The energy difference leads to a phase difference $\Delta \Phi_{V}=2 \pi e V t_{0} / h$, where $t_{0}$ is the ( $B$-dependent) time the reflected and trans- 

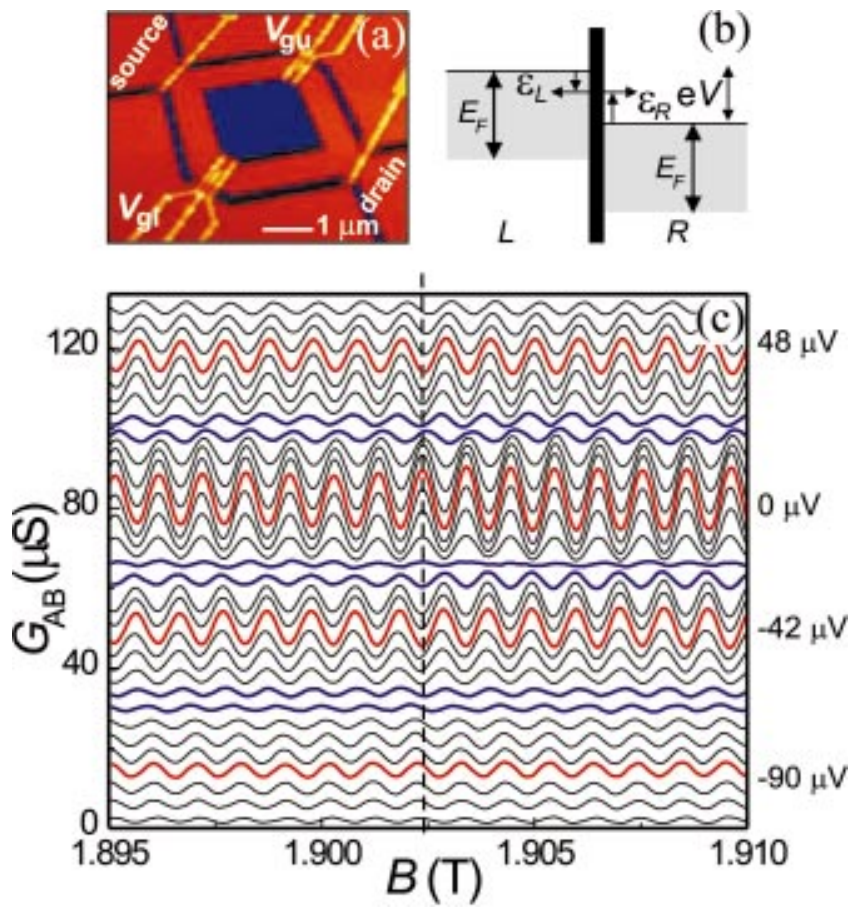

FIG. 1. (Color) (a) Atomic force microscope image of the device. The $\mathrm{AB}$ ring is defined in a $2 \mathrm{DEG}$ by dry etching (dark regions, depth $\sim 75 \mathrm{~nm})$. The 2DEG with electron density 3.4 $\times 10^{15} \mathrm{~m}^{-2}$ is situated $100 \mathrm{~nm}$ below the surface of an $\mathrm{Al}_{x} \mathrm{Ga}_{1-x} \mathrm{As} / \mathrm{GaAs}$ heterostructure (bulk mobility $\mu=7.9$ $\times 10^{5} \mathrm{~cm}^{2} / \mathrm{V} \mathrm{s}$ at $1.5 \mathrm{~K}$ ). In both arms of the ring (lithographic width $0.5 \mu \mathrm{m}$; perimeter $6.6 \mu \mathrm{m}$ ) a barrier can be defined by applying negative voltages to the gate electrodes $V_{g u}$ and $V_{g l}$. The other gates are not used. (b) Schematic energy picture at one of the barriers (see text). (c) Differential $\mathrm{AB}$ conductance, $G_{\mathrm{AB}}$, as a function of $B$ at $15 \mathrm{mK}$ for different source drain voltages, $V$, separated by $5.3 \mu \mathrm{V}$. The traces have a vertical offset. Four values for $V$ are indicated on the right. The dashed line highlights a phase change by a change in the amplitude and sign of the $\mathrm{AB}$ oscillations, indicating an electrostatic $\mathrm{AB}$ effect.

mitted electron waves spend in their respective parts of the ring before they interfere. ${ }^{8}$ We thus expect electrostatic $\mathrm{AB}$ oscillations by changing $V$.

The dashed line in Fig. 1(c) highlights the effect of the applied dc voltage, $V$. The red curve at $V=48 \mu \mathrm{V}$ has a minimum when it crosses the dashed line. By decreasing $V$ the amplitude of the $\mathrm{AB}$ oscillations decreases. The two blue curves near $V=24 \mu \mathrm{V}$ show a case where the amplitudes are small and the sign of the amplitude changes. At $V=0 \mu \mathrm{V}$, the amplitude is maximal. Comparing the 48 and $0 \mu \mathrm{V}$ traces, the $\mathrm{AB}$ oscillation has acquired a change in phase by $\pi$. This continues by another phase shift of $\pi$ when $V$ is decreased to $-42 \mu \mathrm{V}$. Thus, the electrostatic period, $V_{0}$, is $90 \mu \mathrm{V}$ at this magnetic field. This corresponds to an electron travel time $t_{0}=45 \mathrm{ps}$. We note that the Onsager-Büttiker symmetry relation $G(B)=G(-B)$ for a two-terminal measurement ${ }^{14}$ only holds at small bias voltages. Therefore, the phase of the oscillations at the field of the dashed line is not restricted to 0 or $\pi$.

Figure 2 shows a color scale plot of $G_{A B}$ versus $B$ and $V$

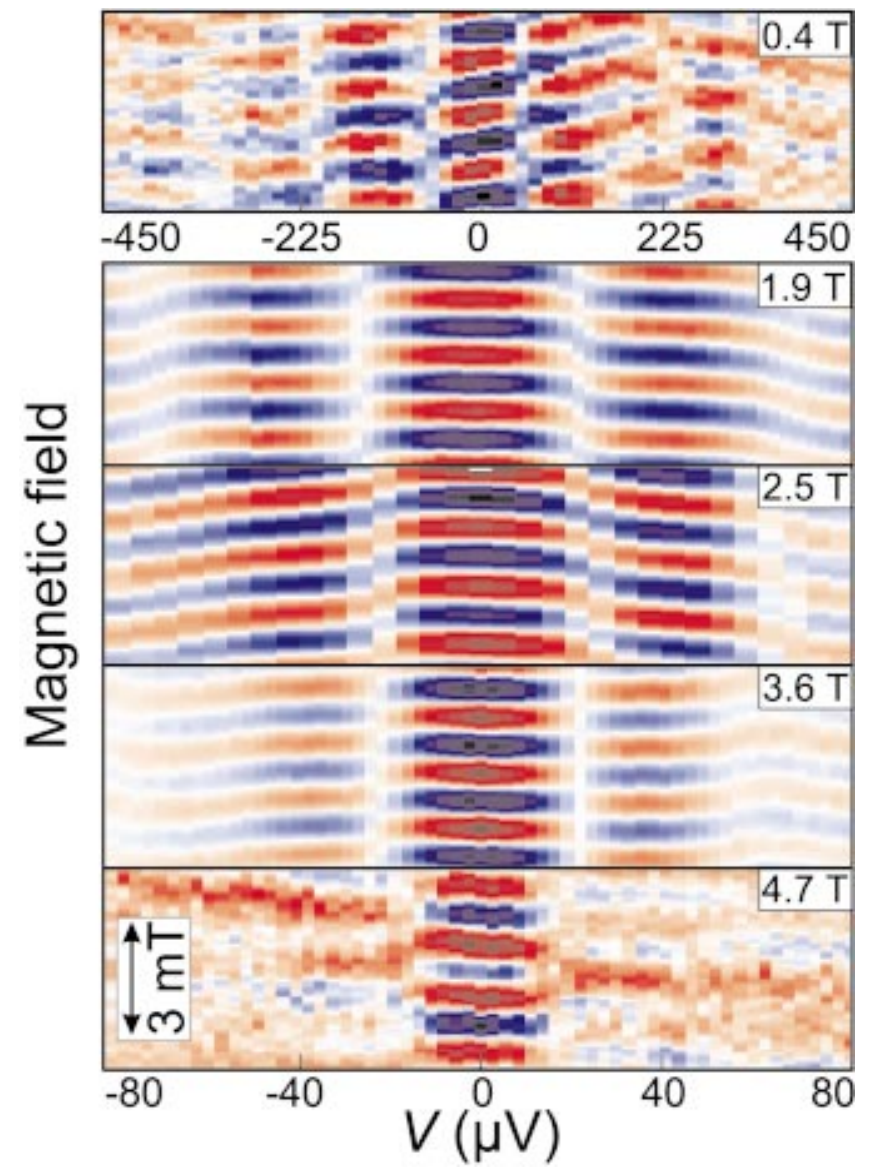

FIG. 2. (Color) Color scale plots of the AB conductance in the plane of magnetic field (vertical axis) around different $B$ values and voltage, $V$ (horizontal axis). Red (blue) corresponds to maximum (minimum) $\mathrm{AB}$ conductance. White corresponds to zero $\mathrm{AB}$ conductance. The phase changes indicate the electrostatic period, which varies from $V_{0}=225 \mu \mathrm{V}$ at $B=0.4 \mathrm{~T}$ to $V_{0}=60 \mu \mathrm{V}$ at $B=4.7 \mathrm{~T}$.

for five different $B$ ranges. The main features of the results are as follows. The amplitude of the $\mathrm{AB}$ oscillations is relatively small, ranging from $\sim 0.01 G$ to $\sim 0.1 G$. The period of the oscillations in $G_{A B}(V)$ decreases with $B$, as is clearly seen by comparing the different panels in Fig. 2. For $B$ $=0.4 \mathrm{~T}, 1.9 \mathrm{~T}, 3.6 \mathrm{~T}$, and, less clearly, for $B=4.7 \mathrm{~T}$, the phase of the magnetic oscillations exhibits sharp $\pi$ shifts at lower voltages. At higher voltages, the phase changes more smoothly. At $B=2.5 \mathrm{~T}$, the phase changes smoothly over the entire voltage range. We return to the phase evolution at different $B$ and $V$ later, when we discuss our scattering model.

The $\mathrm{AB}$ amplitude decreases with $V$ and, the higher $B$, the faster the decrease. The decreasing $\mathrm{AB}$ amplitude is clearly seen in Fig. 3 where we plot the normalized $\mathrm{AB}$ conductance, $G_{A B}(V) / G_{A B}(V=0)$, versus $V$ at $B=1.9 \mathrm{~T}$. We stress that Fig. 3 is obtained by taking a single horizontal cut, i.e., for a single $B$ with no averaging, ${ }^{15}$ from the $B=1.9 \mathrm{~T}$ panel of Fig. 2 (but over a larger voltage range). Note that $G_{A B}(V) / G_{A B}(V=0)$ can be negative since it only represents the oscillating part of the total conductance. The curve is approximately symmetric in $V\left[G_{A B}(V) \approx G_{A B}(-V)\right]$, indi- 


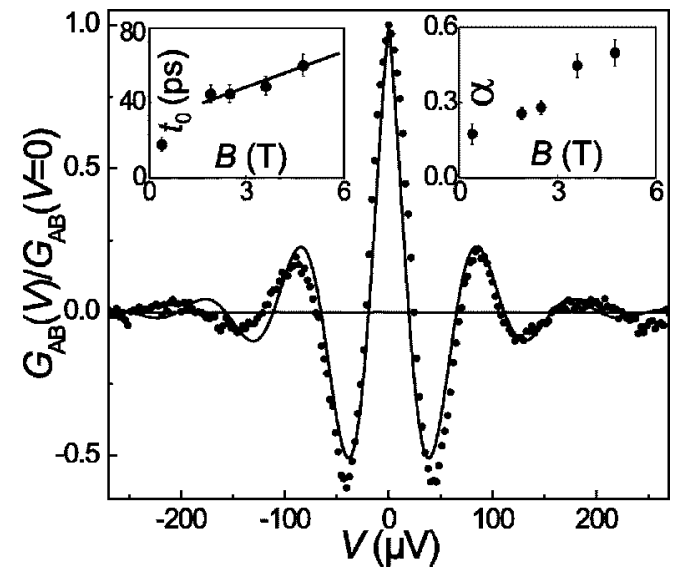

FIG. 3. Normalized $\mathrm{AB}$ conductance $G_{\mathrm{AB}}(V) / G_{\mathrm{AB}}(V=0)$ versus $V$ at $B=1.9 \mathrm{~T}$. The amplitude decreases rapidly with $V$ and, contrary to the magnetic $\mathrm{AB}$ effect, only a few oscillations can be observed. The red curve is a fit with Eq. (4), $\alpha=0.26, t_{0}=45$ ps. The left inset shows the values of the travel time $t_{0}$ versus $B$ as extracted from fits with Eq. (4), varying from 18 ps at $B=0.4 \mathrm{~T}$ to $60 \mathrm{ps}$ at $B=4.7 \mathrm{~T}\left(L / v_{F}=13 \mathrm{ps}\right.$, with $L$ the distance between the barriers and $v_{F}$ the Fermi velocity). The values of $t_{0}$ in the edge channel regime are fitted to a line with a slope of $6 \mathrm{ps} / \mathrm{T}$. The right inset shows the extracted values for $\alpha=\hbar / 2 \varepsilon \tau_{\phi}(\varepsilon)$. The values of $\alpha$ increase with $B$, indicating an increasing importance of manybody effects at quantizing magnetic fields.

cating that within the range of applied voltages the electron travel time, $t_{0}$, hardly changes with $V$. Thus, the electrostatic period does not vary within the applied bias window and we cannot attribute the decrease with $V$ in Fig. 3 to selfaveraging of trajectories with different periods. We therefore believe that the only possible mechanism for the decrease is electron dephasing due to inelastic processes.

The small relative amplitude of the $\mathrm{AB}$ oscillations indicates strong elastic scattering in the arms, whereas the quickly decreasing $\mathrm{AB}$ amplitude with $V$ indicates strong inelastic scattering. Since these scattering mechanisms were not included in the model of Ref. 8, we introduce a simple interference model including dephasing, proceeding along the lines of the general scattering formalism. ${ }^{16}$

We assume single-channel tunnel junctions with transmission amplitudes $t_{1}$ and $t_{2}$, respectively. A small AB amplitude implies that the amplitudes of propagating waves between the junctions, $r_{12}^{L}$ or $r_{21}^{R}$ (see Fig. 4), are small so that we only consider scattering processes of first order in $r$. The oscillating part of the transmission probability, $T_{A B}$, is built up from the pairwise interference of the electron trajectories shown in Fig. 4. Collecting the contributions at a given energy, we obtain

$$
T_{A B}=2 \operatorname{Re}\left[t_{1} t_{2}^{*} e^{i \Phi_{A B}}\left(r_{12}^{L}+r_{21}^{* L}\right)\left(r_{21}^{R}+r_{12}^{* R}\right)\right]
$$

We assume right-left symmetry ${ }^{17}$ and specify the energy dependence of the propagation amplitudes as follows:

$$
r_{12(21)}^{L}=r_{-(+)} e^{i \varepsilon_{L} t_{0} / \hbar}, \quad r_{12(21)}^{R}=r_{+(-)} e^{i \varepsilon_{R} t_{0} / \hbar},
$$

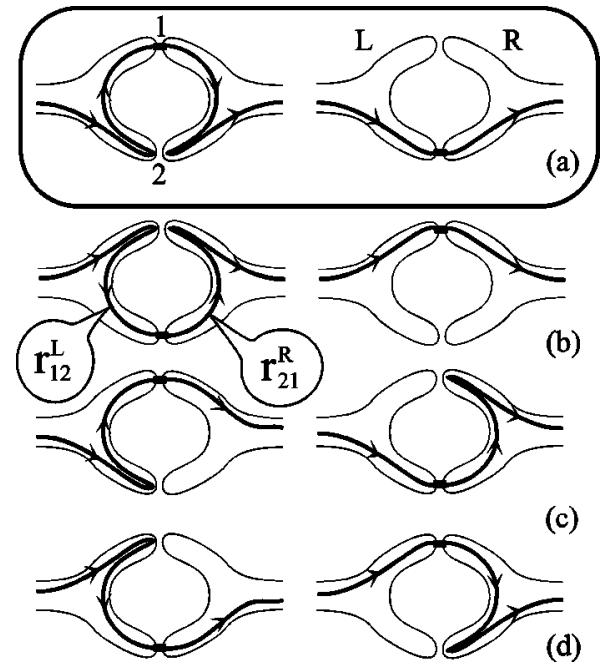

FIG. 4. Schematic diagrams of interfering electron trajectories contributing (in first order) to the oscillating part of the transmission probability. At $B=0$, the pairs of trajectories shown in (a)-(d) contribute equally, whereas at high magnetic field only the pair shown in (a) is relevant (B points downwards).

where $r_{+(-)}$correspond to (counter)clockwise propagation of electrons along the ring (remember that $e V=\varepsilon_{R}-\varepsilon_{L}$ ). For the differential conductance this yields (assuming $t_{1}$ $\left.=t_{2}\right)$

$$
\begin{aligned}
G_{A B}(V) / G= & \left|r_{-}\right|^{2} \cos \left(e V t_{0} / \hbar\right) \cos \left(2 \pi \Phi / \Phi_{0}+\Phi_{a}\right) \\
& +\left|r_{+}\right|^{2} \cos \left(e V t_{0} / \hbar\right) \cos \left(2 \pi \Phi / \Phi_{0}+\Phi_{b}\right) \\
& +2\left|r_{+} r_{-}\right|\left[\cos \left(e V t_{0} / \hbar+\Phi_{c}\right)+\sin \left(e V t_{0} / \hbar\right.\right. \\
& \left.\left.+\Phi_{c}\right) e V t_{0} / \hbar\right] \cos \left(2 \pi \Phi / \Phi_{0}+\Phi_{d}\right),
\end{aligned}
$$

where $\Phi_{a, b, c, d}$ are contributions to the phase that vary slowly with $B$ in comparison to $\Phi / \Phi_{0}$ (e.g., due to bending of the electron trajectories). At small $B$ time reversability holds, implying $r_{+}=r_{-}$and $\Phi_{a, b, d}=0$. Under these conditions, the phase of the magnetic oscillations only changes by $\pi$ as a function of $V$. Interestingly, the same happens at high $B$. In this case Lorentz bending suppresses counterclockwise propagation, so that $r_{+} \gg r_{-}$. The result is then dominated by the second term in Eq. (3). For a $B$ interval in which $\Phi_{b}$ can be considered constant, we find again that the phase of the magnetic oscillations only changes by $\pi$ as a function of $V$. In the intermediate regime $r_{+} \simeq r_{-}$, and $\Phi_{a, b, c, d}$ vary slowly with $B$. The phase of the magnetic oscillations in this case continuously shifts with voltage, saturating at $V$ $\gg \hbar / t_{0} e$.

To model electron dephasing due to inelastic processes, we assume that the electron amplitudes at a given energy are suppressed by a factor $\exp \left(-t / 2 \tau_{\varphi}\right), \tau_{\varphi}(\varepsilon)$ being the energy-dependent dephasing time. In analogy with results found for a disordered electron gas in the quantum Hall regime, ${ }^{18}$ disordered metal-like systems, ${ }^{19}$ composite fermions, ${ }^{20}$ and a Luttinger liquid, ${ }^{21}$ we propose a dephasing time proportional to energy, $\hbar / \tau_{\varphi}(\varepsilon)=2 \alpha \varepsilon$. Here $\alpha$ is a dimensionless factor of the order of the dimensionless $2 \mathrm{DEG}$ 
conductance, $G /\left(e^{2} / h\right)$. A small value for $\alpha(\alpha \ll 1)$ and large conductances $\left(G \gg e^{2} / h\right)$ correspond to vanishing electron-electron interactions. ${ }^{19}$ In contrast, $\alpha \simeq 1$ and $G$ $\simeq e^{2} / h$ signal the importance of many-body effects. In the limit of $r_{+} \gg r_{-}$our model for the dephasing gives

$$
\begin{aligned}
G_{A B}(V) / G= & \left|r_{+}\right|^{2}\left[\cos \left(e V t_{0} / \hbar\right)\right. \\
& \left.-\alpha \sin \left(e|V| t_{0} / \hbar\right)\right] e^{-\alpha e|V| / \hbar} \\
& \times \cos \left(2 \pi \Phi / \Phi_{0}+\Phi_{b}\right) .
\end{aligned}
$$

Our scattering model provides an explanation for the observed experimental results. The model accounts for the abrupt $\pi$-phase changes observed in Fig. 2 at the lowest ( $B$ $=0.4 \mathrm{~T}$ and $1.9 \mathrm{~T})$ and highest $(B=3.6 \mathrm{~T}$ and $4.7 \mathrm{~T}) \mathrm{mag}$ netic fields (provided $V$ is small). In the intermediate regime $(B=2.5 \mathrm{~T})$ the model explains also why the phase varies more continuously. The well-defined period $t_{0}$ probably indicates the formation of an edge channel connecting the junctions. However, the magnitude of the $\mathrm{AB}$ oscillations is small. This signals a strong scattering to and from the edge channel involving almost localized states at higher magnetic field. One can estimate $\left|r_{+}\right|^{2}$ as a classical probability, assuming uniform distribution over the transport channels in the ring and $1 / 3$ suppression due to scattering near the openings to the source and drain leads. This gives $\left|r_{+}\right|^{2} \simeq \frac{1}{3} N_{\text {tr }}$ $=0.03$, which is in agreement with the experimental value of $G_{\mathrm{AB}}(V=0) / G \simeq 0.02$ at low $B$.

We use Eq. (4) to fit the experimental $V$ dependence of the $\mathrm{AB}$ amplitude, as shown in Fig. 3 for the particular case $B$ $=1.9 \mathrm{~T}$. The reasonable quality of the fit supports our model for the dephasing time. From such fits we extract values for $t_{0}$ and $\alpha$ at various magnetic fields. The left inset to Fig. 3 shows our results for the $B$ dependence of $t_{0}$. The values in the edge channel regime are fitted to a straight line with a slope of $6 \mathrm{ps} / \mathrm{T}$. The increase of the travel time can be attributed to the decreasing drift velocity, $v_{\text {drift }}=|\mathbf{E}| / B,|\mathbf{E}|$ being the modulus of the confining electric field at the arm edges. An estimate based on parabolic confinement gives $|\mathbf{E}|$ $\simeq(1-4) \times 10^{5} \mathrm{~V} / \mathrm{m}$. This implies a slope $d t_{0} / d B$ between 5 and $20 \mathrm{ps} / \mathrm{T}$, which is in reasonable agreement with the observed value.

In the right inset to Fig. 3 we show our results for $\alpha$ $=\hbar / 2 \varepsilon \tau_{\phi}(\varepsilon)$. The $\alpha$ values are rather high and increase with $B$. At $\varepsilon=10 \mu \mathrm{eV}$, we find $\tau_{\phi}=180 \mathrm{ps}$ at $B=0.4 \mathrm{~T}$ and $\tau_{\phi}$ $=65$ ps at $B=4.7 \mathrm{~T}$. The usual Fermi-liquid theory assumes well-defined quasiparticles, corresponding to $\alpha \ll 1$. The fact that we observe $\alpha \simeq 1$ signals the importance of many-body effects (electron-electron interactions). The Fermi-liquid theory here is on the edge of applicability. We attribute this to significant scattering in the arms of the ring. For a clean 2DEG one expects no significant many-body effects until $\nu$ $<1\left(B_{\nu=1}=14 \mathrm{~T}\right.$ in our 2DEG). One can consider $\alpha$ as an effective dissipative conductance in units of $e^{2} / h \cdot{ }^{18,19}$ In our case, the relevant conductance is that of the arms of the $\mathrm{AB}$ ring, which is of the order of $e^{2} / h$ at the highest magnetic fields. This is consistent with the observed $\alpha$ values.

In conclusion, using an electromagnetic $\mathrm{AB}$ effect, we find a method to determine the nonequilibrium electron dephasing time in a 2DEG, which becomes very short at high voltages and magnetic field.

We thank G. Seelig, M. Büttiker, T. Hayashi, Y. Hirayama, A. van Oudenaarden, and R. Schouten for their help. We acknowledge financial support from the DARPA Grant No. DAAD19-01-1-0659 of the QuIST program; the Specially Promoted Research Grant-in-Aid for Scientific Research; the Ministry of Education, Science and Culture in Japan; the Dutch Organization for Fundamental Research on Matter; the New Energy and Industrial Technology Development Organization Joint Research Program (NTDP-98).
*Also at ERATO Mesoscopic Correlation Project.

${ }^{1}$ Y. Aharonov and D. Bohm, Phys. Rev. 115, 485 (1959).

${ }^{2}$ R.A. Webb et al., Phys. Rev. Lett. 54, 2696 (1985).

${ }^{3}$ G. Timp et al., Phys. Rev. Lett. 58, 2814 (1987).

${ }^{4}$ J.C. Licini et al., Phys. Rev. Lett. 55, 2987 (1985).

${ }^{5}$ S. Washburn et al., Phys. Rev. Lett. 59, 1791 (1987).

${ }^{6}$ P.G.N. de Vegvar et al., Phys. Rev. B 40, R3491 (1989).

${ }^{7}$ B. Krafft, A. Förster, A. van der Hart, and Th. Schäpers, Physica E (Amsterdam) 9, 635 (2001).

${ }^{8}$ Yu.V. Nazarov, Phys. Rev. B 47, 2768 (1993); Physica B 189, 57 (1993).

${ }^{9}$ A. van Oudenaarden et al., Nature (London) 391, 768 (1998).

${ }^{10}$ P. Mohanty, E.M.Q. Jariwala, and R.A. Webb, Phys. Rev. Lett. 78, 3366 (1997).

${ }^{11}$ T. Schmidt et al., Phys. Rev. Lett. 86, 276 (2001).

${ }^{12}$ J. Könemann et al., Phys. Rev. B 64, 155314 (2001).

${ }^{13}$ W.G. van der Wiel et al., Science 289, 2105 (2000).

${ }^{14}$ M. Büttiker, IBM J. Res. Dev. 32, 317 (1988).

${ }^{15}$ In Ref. 9 the correlation function $\left\langle G_{\mathrm{AB}}(B, V) G_{\mathrm{AB}}(B+\Delta B, V\right.$ $+\Delta V)\rangle$ is calculated, where the angle brackets denote an en- semble average. For a given $\Delta V$, one averages over an ensemble of pairs of $G_{\mathrm{AB}}-B$ traces, each pair having a $\Delta V$ voltage difference. This recipe has the effect that, if the potential landscape fluctuates on the scale of $\Delta V$, and hence affects the (electrostatic) $\mathrm{AB}$ period, the correlation function decreases.

${ }^{16}$ M. Büttiker, Phys. Rev. Lett. 57, 1761 (1986).

${ }^{17}$ This is a reasonable assumption, since in our experiment we tune the barriers to have equal transmission.

${ }^{18}$ D.G. Polyakov and K.V. Samokhin, Phys. Rev. Lett. 80, 1509 (1998).

${ }^{19}$ B.L. Altshuler and A.G. Aronov, in Electron-Electron Interactions in Disordered Systems, edited by A. L. Efros and M. Pollak (North-Holland, Amsterdam, 1985).

${ }^{20}$ P.A. Lee, E.R. Mucciolo, and H. Smith, Phys. Rev. B 54, 8782 (1996).

${ }^{21}$ M.P.A. Fisher and L.I. Glazman, in Mesoscopic Electron Transport, edited by L.L. Sohn, L.P. Kouwenhoven, and G. Schön, NATO ASI, Ser. E (Kluwer, Dordrecht, 1997), Vol. 345, pp. 331-373. 